\title{
Clinical Figure and Rate of Mortality among Chronic Schizophrenics: A Local Assessment
}

\section{Saeed Shoja Shafti, Alireza Memarie, Behjat Rahimi, Masomeh Rezaie}

University of Social Welfare and Rehabilitation Sciences (USWR) Razi Psychiatric Hospital, Iran

*Corresponding Author: Saeed Shoja Shafti, University of Social Welfare and Rehabilitation Sciences (USWR) Razi Psychiatric

Hospital, Iran

Received date: February 15, 2020; Accepted date: February 18, 2020; Published date: February $24,2020$.

Citation: Saeed S Shafti, Alireza M, Behjat R, Masomeh R (2020) Clinical Figure and Rate of Mortality Among Chronic Schizophrenics: A Local Assessment, 2(2): DOI: 10.31579/2690-8794/013

Copyright: ( 2020 Saeed S Shafti, This is an open-access article distributed under the terms of The Creative Commons Attribution License, which permits unrestricted use, distribution, and reproduction in any medium, provided the original author and source are credited.

\section{Abstract:}

Introduction: Researchers have consistently reported that people with mental disorders have elevated mortality compared with the general population. In Iran there are not systematic psychiatric case registers that could allow us to study precisely the mortality of psychiatric patients. The aim of the current study was to determine the mortality rate and clinical profile of death in a group of non-western chronic elderly schizophrenic patients.

Methods: chronic geriatric subdivision of Razi Psychiatric hospital with a capacity around 220 beds (110 for each of male and female elderly patients) had been selected as the specific arena of investigation. For the present retrospective survey, all recorded deceases during the last sixty months (April of 2014-August 2019) in the said senior wards had been included in the current study. Clinical diagnosis, too, was essentially based on 'Diagnostic and Statistical Manual of Mental Disorders', $5^{\text {th }}$ edition (DSM-5).

Results: Among eight-hundreds and frothy chronic elderly schizophrenic patients, sixty-nine deceases had been registered by the mortality committee of the hospital. As said by results, the annual rate of mortality among elderly schizophrenic patients in the present assessment was around 0.015 ( 0.15 per 1,000 individuals per year) and 0.017 (0.17 per 1,000 individuals per year) among male and female aged patients, respectively, which were significantly lower than current native crude death rate $(p<0.000)$. While the age of the expired female patients was significantly more than the died male schizophrenics $(\mathrm{p}<0.001)$, the life expectancy of both male and female expired patients was significantly shorter than the public's life expectancy ( $<<0.000)$. Besides, in the present evaluation, while cardiac illness was the main leading cause of death among old schizophrenics, reasons like suicide, falls, drug use or tuberculosis, were not applicable at all.

Conclusion: While the rate of mortality among elderly schizophrenics was significantly lower than public's crude death rate, age of the deceased female patients was significantly longer than the male expired patients and life expectancy of both male and female died patients was significantly lower than native public's life expectancy. Cardiac disorder, as well, was the main leading cause of death among aged schizophrenic patients.

Key Words: schizophrenia; rate of mortality; geriatric psychiatry; life expectancy; cause of death; chronic mental patients.

\section{Introduction:}

Researchers have consistently reported that people with mental disorders have elevated mortality compared with the general population $[1,2]$. Since then, numerous studies and reviews have been conducted on the mortality risks of people with a variety of mental disorders and specific diagnoses [3, 4]. The link between mental disorders and mortality is complicated because most people with mental disorders do not die of their condition; rather, they die of heart disease and other chronic diseases, infections, suicide, and other causes [5, 6]. Another complicating factor is that mental disorders are associated with risk factors for mortality [7]. In this regard, people with mental disorders have high rates of adverse health behaviors, including tobacco smoking, substance use, physical inactivity, and poor diet[7]. In turn, these behaviors contribute to the high rates of chronic medical conditions among people with mental disorders

[8]. Although schizophrenia is not in itself a lethal illness, over-mortality among such group of psychiatric patients in comparison to the reference population has been attested to for a long time [9]. Until the use of antibiotics, this over-mortality was mainly due to infectious diseases caused by the close quarters in mental institutions. At the present time this over-mortality is mainly due to suicide but also with a noteworthy mortality by certain natural causes such as respiratory diseases and cardio-vascular and cerebro-vascular diseases [9]. On the other hand, old age, or late adulthood, usually refers to the stage of the life cycle that begins at age 65. Older adults can also be described as well-old (persons who are healthy) and sick-old (persons who have an infirmity that interferes with functioning and requires medical or psychiatric attention) [10]. The health needs of older adults have grown enormously as the population ages, and geriatric physicians and psychiatrists play major roles in treating this population [10]. While the leading causes of death among older persons are heart disease, cancer, and stroke [11], the National Institute of Mental Health's Epidemiologic Catchment Area (ECA) program has found that the most common mental disorders of old age are depressive disorders, cognitive disorders, phobias, and alcohol use disorders. Older adults also have a high risk for suicide and drug-induced psychiatric symptoms [12]. Meanwhile, elderly persons have a higher risk for suicide than any other population [13]. In this regard, some scholars believe that mortality is significantly higher among people with mental disorders than among the comparison population and mental disorders rank among the most substantial causes of death worldwide [1], and mortality risk of the long-stay psychiatric patients compared with that of the general population was 
notably higher, despite ongoing improvements in medical care and facilities $[14,15.16]$. But there are other scholars as well, who believe that standardized mortality ratios are lowest in geriatric psychiatric patients [17] and the ostensible over-mortality tends to disappear in elderly subjects [18]. Also with regard to leading causes of death, while some of researchers believe that long-stay psychiatric patients were found to die from the same natural causes as the rest of the general population $[11,16]$, others accentuate on more specific causes $[19,20]$. In Iran there are not systematic psychiatric case registers that could allow us to study precisely the mortality of psychiatric patients. So, the aim of the current study was to determine the mortality rate and clinical profile of death in a group of non-western chronic elderly schizophrenic patients

\section{Methods:}

Razi psychiatric hospital in south of capital city of Tehran, as one of the largest and oldest public psychiatric hospitals in the Middle East, which has been established formally in 1917 and with a capacity around 1375 active beds, had been selected as the field of study in the present retrospective assessment. Moreover, amongst its separate existent sections, chronic geriatric subdivision of the hospital with a capacity around 220 beds (110 for each of male and female elderly patients) had been selected as the specific arena of investigation. So for survey, all recorded deceases during the last sixty months (April of 2014-August 2019) in the said senior wards had been included in the current study. Clinical diagnosis, too, was essentially based on 'Diagnostic and Statistical Manual of Mental Disorders', $5^{\text {th }}$ edition (DSM-5) [21].

\section{Statistical analysis:}

't-test' and 'comparison of proportions' had been used as arithmetic formularies for valuation of data. Statistical significance, as well, had been defined as $p$ value $\leq 0.05$. MedCalc Statistical Software version 15.2 was used as statistical software tool for analysis.

\section{Results:}

Among eight-hundreds and frothy elderly schizophrenic patients hospitalized in the chronic geriatric section of Razi psychiatric hospital ( $n=505 \& n=335$, for male and female aged patients, respectively), during the last sixty-four months, sixty-nine deceases (thirty-nine deaths among male patients, and thirty expiries among female patients) had been registered by the mortality committee of the hospital. As said by results, the annual rate of mortality among elderly psychiatric patients in the present assessment was around 0.015 (0.15 per 1,000 individuals per year) and 0.017 ( 0.17 per 1,000 individuals per year) among male and female elderly patients, respectively, which could not show any significant gender-based difference, incidentally $(\mathrm{z}=-0.6370, \mathrm{p}-<0.52$, CI $95 \%=-0.05,0.02)$. Nevertheless, they were significantly lower than the current native crude death rate (4.84 deaths / 1000 population) $(\mathrm{z}=6.09, \mathrm{p}<0.000, \mathrm{CI} 95 \%=0.005,0.002)$. On the other hand, the mean \pm standard deviation (SD) of the age of the deceased patients were around $68.2 \pm 6.81$ and $73.57 \pm 6.82$ for male \& female patients, respectively, which could well reveal a significant gender-based difference, by the way $(\mathrm{t}=3.245$, probability $<0.001$, CI $95 \%=2.07,8.67)$. Furthermore, in comparison with the life expectancy in Iran, based on the latest WHO data published in 2018, which was around 74.6 and 76.9 for male \& female persons, respectively, and 75.7, as total, findings of the present assessment displayed a significant shortening of life among both male and female aged patients $(\mathrm{t}=21.11, \mathrm{p}<0.000$, CI 95\% $=5.81,6.99$, and $\mathrm{t}=8.93, \mathrm{p}<0.000$, CI $95 \%=2.60,4.06$, respectively). With regard to leading causes of death, results of the present assessment had exposed that cardiac disorders, in total, was the main leading cause of death among aged schizophrenic patients (Table 1). Regarding other causes of death, like stroke, lower respiratory infections, chronic obstructive pulmonary disease, and malignancy, the present ranking was rather roughly in concurrence with the national or universal data, maybe due to preventable problems like electrolyte imbalance or aspiration । asphyxiation, which demands more medical cautiousness or monitoring. Moreover, with regard to malignancy, trachea, bronchus or lung cancers was evident in only two male cases, and the rest included stomach, pancreas and brain cancers, as well as lymphoma. Likewise, causes like violence, suicide, road traffic accidents, falls, fires, drug use, tuberculosis, drowning, epilepsy, and Parkinson's disease were not applicable at all, in the present evaluation.

\section{Discussion:}

While mental disorders are supposed to be associated with an elevated risk of premature mortality [1], inadequately organized somatic care and the prevailing culture of "non-somatic" treatment in psychiatry were suggested to, at least in part, explain this phenomenon [16]. Such a problem, also, maybe can be exacerbated by the shift in psychiatric care from inpatient facilities to community settings, jails, and prisons in recent decades [22]. Evidence from national registry studies indicates that this mortality risk is especially high soon after discharge from inpatient psychiatric services [23, 24]. Elderly patients have more concomitant, chronic, and multiple medical problems and take more medications than younger adults [25]; many of these medications can influence their mental status [26]. Back to our discussion and with respect to the mortality rate, our conclusion was somewhat in accord with the outcomes of Ran et al., who supposed that standardized mortality ratios were highest in young subjects and the lowest in geriatric subjects [17]. But, instead, it was not in agreement with the inferences of Lim et al. [14], Hewer et al. [15], and Räsänen et al. [16], who assumed that the mortality risk of the long-stay psychiatric patients compared with that of the general population was notably higher. Furthermore, it was in partial agreement with Bralet et al., who said that though the average mortality in schizophrenia is twice higher than among the population, this over-mortality is highest among the 20-40 years range of age and added risk tends to disappear after 60 years (18). So the reports of Walker et al. [1] that mortality is significantly higher among people with mental disorders than among the comparison population and mental disorders rank among the most substantial causes of death worldwide may demand reconsideration. Also, while in the present assessment and with respect to mortality rate there was not any meaningful difference between old male and female psychiatric patients, this finding was not in harmony with the deduction of Tabbane et al., who stated that the supposed over-mortality concerned only schizophrenic male patients whereas schizophrenic females did not have an over-mortality [19]. In addition, while a substantial difference was evident between male and female old patients as regards the age of death, which was significantly longer on behalf of female patients, the latter finding is coherent with the fact that on average, women live longer than men [11], and may highlight some kind of biological vulnerability, which demands further gender-based clinical investigations. Moreover, with respect to gender-based mortality rate, our verdict was not compatible with the assumptions of Gausset et al. [27], who supposed that among severe mental patients, mortality rates are higher for men than women. Similarly, as regards life expectancy, outcomes of the current evaluation, which showed a significant shortening of life among elderly male and female schizophrenic patients, were consistent with the findings of Zgueb et al. [28], who stated that young psychiatric inpatients seem to be at high risk of premature death and Zubenko et al. [29], who supposed that the mental disorders of late life have a significant negative impact on the survival of older psychiatric patients. With regard to leading causes of death, results of the present assessment had exposed that cardiac disorders, in total, was the main leading cause of death among geriatric patients, which was in agreement with the conclusions of Trollor et al. 
(11) and Räsänen et al. [16], who believed that long-stay psychiatric patients were found to die from the same natural causes as the rest of the general population. Regarding other causes of death, the present ranking was rather roughly in concurrence with the findings of Lim et al. [14], Hewer et al. [15], Tabbane et al.[19], and Abiodun OA [20]. Such a variance maybe is a bit due to preventable problems like electrolyte imbalance or aspiration \ asphyxiation, which had occupied the second ranking, among all causes of death and demands more medical cautiousness or monitoring. Also, with respect to infection and pneumonia, our results were not consistent with the assumptions of Lim et al. (14), and Abiodun OA [20], who had declared, in general, infection and pneumonia as the most important causes of death. The later finding, perhaps, could be attributed to proper scrubbing and ventilation, which may well reduce the risk of infections. But, regarding sudden cardiac death our conclusion was somewhat compatible with the finding of Abiodun OA, who had found sudden unexplained deaths in a noticeable number of cases (20). Also, in opposite to Walker et al. [1] and Bralet et al. [18], unnatural causes, like suicide, did not have any place among main causes of death in our study. Nonetheless, as regards the role of malignancy, it was rather in harmony with the statement of Tabbane et al., who thought that cancer mortality in schizophrenic patients is still debated [19]. In general, at all times a pretreatment medical evaluation, plus periodic exams, based on standard protocols, is essential [30]. For example, any changes in blood pressure and pulse rate and other side effects should be watched [31]. Attention ought to increasingly focus on somatic examinations and various health educational programs specially designed for psychiatric patients and involving matters like healthy diet, smoking cessation and physical exercise [32]. Anyhow, the importance of mortality studies remains twofold: they are a good indicator of the quality of health care policy, and also they enable the formulation of research hypotheses to be made if you point out specific causes of death in a subgroup like schizophrenics compared to the general population [33]. For patients hospitalized with psychiatric disorders, the time shortly after discharge is the period in which they are at the highest risk for premature death from a variety of causes. Clinicians should keep these patients safe by serving as a liaison between primary and secondary health care services to ensure patients are receiving holistic care that meets their physical and mental health needs as well as addresses their psychosocial problems. Mental health facilities and their partner agencies need to work proactively and in unison to determine the risks likely to reemerge or become exacerbated at discharge and to provide extra support to ameliorate the greatly elevated risk of unnatural death [22]. Identifying risk factors for deaths in psychiatric hospitals highlights needed changes in psychiatric management strategies taking into account the patient's characteristics as well as the drugs' safety profile. Further studies with larger samples are needed to better highlight risk factors for premature death in psychiatric inpatients. Identifying such risk factors is necessary to develop efficient preventive strategies [28].

\section{Conclusion:}

While the rate of mortality among elderly schizophrenics was significantly lower than public's crude death rate, age of the deceased female patients was significantly longer than the male expired patients and life expectancy of both male and female died patients was significantly lower than native public's life expectancy. Cardiac disorder, as well, was the main leading cause of death among aged schizophrenic patients.

\begin{tabular}{|c|c|c|c|c|c|}
\hline Comparative Ranking & Cause of death & Male & Female & Total & $\%$ \\
\hline $\mathbf{1}$ & Myocardial infarction & 4 & 5 & 9 & 13.04 \\
\hline $\mathbf{2}$ & Aspiration and asphyxiation & 4 & 4 & 8 & 11.59 \\
\hline $\mathbf{2}$ & Electrolyte imbalance & 5 & 3 & 8 & 11.59 \\
\hline $\mathbf{3}$ & Cancer & 4 & 3 & 7 & 10.14 \\
\hline $\mathbf{3}$ & Sudden Cardiac death & 3 & 4 & 7 & 10.14 \\
\hline $\mathbf{4}$ & Respiratory failure (COPD) & 4 & 2 & 6 & 8.69 \\
\hline $\mathbf{4}$ & Septicemia & 2 & 4 & 6 & 8.69 \\
\hline $\mathbf{5}$ & Cerebrovascular accident & 4 & 1 & 5 & 7.24 \\
\hline $\mathbf{5}$ & Congestive heart failure & 3 & 2 & 5 & 7.24 \\
\hline $\mathbf{6}$ & Pneumonia & 3 & 1 & 4 & 5.79 \\
\hline $\mathbf{7}$ & Renal failure & 2 & 0 & 2 & 2.89 \\
\hline $\mathbf{8}$ & Pulmonary thromboembolism & 1 & 0 & 1 & 1.44 \\
\hline $\mathbf{8}$ & Encephalitis & 0 & 1 & 1 & 1.44 \\
\hline
\end{tabular}

\section{References:}

1. Walker ER, McGee RE, Druss BG.(2015) Mortality in Mental Disorders and Global Disease Burden Implications A Systematic Review and Meta-analysis JAMA Psychiatry ; 72(4):334-341.

2. Harris EC, Barraclough B. ( 1998) Excess mortality of mental disorder. Br J Psychiatry; 173:11-53.

3. Lawrence D, Kisely S, Pais J.(2010) The epidemiology of excess mortality in people with mental illness. Can J Psychiatry; 55(12):752-760.

4. Chesney E, Goodwin GM, Fazel S. (2014) Risks of all-cause and suicide mortality in mental disorders: a meta-review. World Psychiatry; 13(2):153-160.
5. Saha S, Chant D, McGrath J.(2007) A systematic review of mortality in schizophrenia: is the differential mortality gap worsening over time? Arch Gen Psychiatry; 64 (10): 1123-1131.

6. Cuijpers P, Vogelzangs N, Twisk J, Kleiboer A, Li J, et al (2014) Comprehensive meta-analysis of excess mortality in depression in the general community versus patients with specific illnesses. Am J Psychiatry; 171(4):453-462.

7. Colton CW, Manderscheid RW. (2006) Congruencies in increased mortality rates, years of potential life lost, and causes of death among public mental health clients in eight states. Prev Chronic Dis; 3(2): 42.

8. Whiteford HA, Degenhardt L, Rehm J (2010.) Global burden of disease attributable to mental and substance use disorders: 
findings from the Global Burden of Disease Study Lancet 2013; 382(9904): 1575-1586.

9. Laursen TM, Nordentoft M, Mortensen PB.(2014) Excess early mortality in schizophrenia. Annu Rev Clin Psychol; 10: 425-448.

10. Balzer DG, Steffens DC. (2012)Essentials of Geriatric Psychiatry. 2nd ed. Arlington: American Psychiatric Association.

11. Trollor JN, Anderson TM, Sachdev PS, Brodaty H, Andrews G.(2007) Prevalence of mental disorders in the elderly: The Australian National Mental Health and Well-Being Survey. Am J Geriatr Psychiatry .5(6): 455-466.

12. Bartels SJ, Naslund JA. (2013) The underside of the silver tsunami-older adults and mental health care. N Engl J Med; 368:493.

13. Kessler RC, Berglund P, Demler O, Jin R, Merikangas KR, et al (2005) Lifetime prevalence and age-of-onset distributions of DSM-IV disorders in the National Comorbidity Survey Replication. Arch Gen Psychiatry; 62(6):593-602.

14. Lim LC, Sim LP, Chiam PC.( 1991) Mortality among psychiatric inpatients in Singapore. Singapore Med J ;32 (3):130-132.

15. Hewer W, Rössler W. (1997) [Mortality of patients with functional psychiatric illnesses during inpatient treatment]. Fortschr Neurol Psychiatr ; 65(4):171-181.

16. 16) Räsänen S, Hakko H, Viilo K, Meyer-Rochow VB, (2003) Moring J. Excess mortality among long-stay psychiatric patients in Northern Finland. Soc Psychiatry Psychiatr Epidemiol ; 38(6): 297-304

17. Ran MS, Chan CL, Chen EY, Tang CP, Lin FR et al (2008) Mortality of geriatric and younger patients with schizophrenia in the community. Suicide Life Threat Behav ; 38(2):143-151.

18. Bralet MC, Yon V, Loas G, Noisette C.(2000) Cause of mortality in schizophrenic patients: prospective study of years of a cohort of 150 chronic schizophrenic patients]. Encephale. ; 26(6): 32-41.

19. Tabbane K, Joober R, Spadone C, Poirier MF, Olié JP(1993) Mortality and cause of death in schizophrenia. Review of the literature]. Encephale ;19(1): 23-28.

20. Abiodun OA. (1988)Mortality in a psychiatric population: a Nigerian psychiatric hospital experience. Acta Psychiatr Scand ; 77(6): 654-657.

21. American Psychiatric Association (APA). Diagnostic and Statistical Manual of Mental Disorders. 5th ed. Washington, DC: American Psychiatric Association, 2013.
Lamb HR, Weinberger LE.( 2005) The shift of psychiatric inpatient care from hospitals to jails and prisons. J Am Acad Psychiatry Law; 33 (4): 529-534.

23. Crump C, Ioannidis JP, Sundquist K, Winkleby MA, Sundquist J. (2013) Mortality in persons with mental disorders is substantially overestimated using inpatient psychiatric diagnoses. J Psychiatr Res; 47(10):1298-1303.

24. Meloni D, Miccinesi G, Bencini A, Conte M, Crocetti E, et al. (2006) Mortality among discharged psychiatric patients in Florence, Italy. Psychiatr Serv.; 57 (10): 1474-1481.

25. Thakur ME, Blazer DG, Steffens DC, eds.(2014) Clinical Manual of Geriatric Psychiatry. Arlington: American Psychiatric Publishing.

26. Reifler BY, Colenda CC, Juul D. (2012) Geriatric psychiatry. In: Aminoff MJ, Faulkner LR, eds. The American Board of Psychiatry and Neurology: Looking Back and Moving Ahead. Arlington: American Psychiatric Publishing;135.

27. Gausset MF, Casadebaig F, Guillaud-Bataille JM, Quemada N, Terra JL. (1992) Mortality of mentally ill patients. Review of the literature. Encephale ; 18 (1): 93-100.

28. Zgueb Y, Jomli R, Ouertani A, Hechmi S, Ouanes S, et al (2014) Deaths in a Tunisian psychiatric hospital: an eleven-year retrospective study. Encephale ; 40(5):416-422.

29. Zubenko GS, Mulsant BH, Sweet RA, Pasternak RE, Tu XM (1997)

30. Mortality of elderly patients with psychiatric disorders. Am J Psychiatry ;154(10):1360-13608.

31. Byers AL, Yaffe K, Covinsky KE, Friedman MB, Bruce ML (2010) High occurrence of mood and anxiety disorders among older adults: The National Comorbidity Survey Replication. Arch Gen Psychiatry; 67(5):489-496.

32. Balzer DG, Steffens DC.(2012) Essentials of Geriatric Psychiatry. 2nd ed. Arlington: American Psychiatric Association.

33. Meesters P, de Haan L, Comijs HC, Max L Stek Maureen M J Smeets-Janssen et al.(2012) Schizophrenia spectrum disorders in later life: Prevalence and distribution of age at onset and sex in a Dutch catchment area. Am J Geriatr Psychiatry; 20 (1):18-28.

34. Casadebaig F, Philippe A. (1999) Mortality in schizophrenic patients. 3 years follow-up of a cohort]. Encephale;25 (4): 329337.

22 . 